\title{
RISK ANALYSIS IN CONSTRUCTION PROJECTS: A PRACTICAL SELECTION METHODOLOGY
}

\author{
${ }^{1}$ Alberto De Marco and ${ }^{2}$ Muhammad Jamaluddin Thaheem \\ ${ }^{1}$ Department of Management and Production Engineering, Politecnico di Torino, Turin, Italy \\ ${ }^{2}$ Department of Urban Studies and Planning, Politecnico di Torino, Turin, Italy
}

Received 2013-06-27; Revised 2013-08-30; Accepted 2013-11-28

\begin{abstract}
Project Risk Management (PRM) is gaining attention from researchers and practitioners in the form of sophisticated tools and techniques to help construction managers perform risk management. However, the large variety of techniques has made selecting an appropriate solution a complex and risky task in itself. Accordingly, this study proposes a practical framework methodology to assist construction project managers and practitioners in choosing a suitable risk analysis technique based on select project drivers. Additionally, the methodology transforms the traditional triple constraints by broadening the focus from the project to a combination of the project and PM organization. Scale harmonization is achieved by dividing the selected project drivers and risk analysis categories into four levels. The applicability and efficiency of the methodology is demonstrated in two actual construction projects by creating a radar chart and performing their ex-post risk analysis with the help of the developed technique. The study contributes to the existing body of knowledge on PRM as a practical tool that helps project managers select suitable risk analysis techniques under given project characteristics.
\end{abstract}

Keywords: Project Risk Management, Construction Management, Decision Making, Project Classification, Risk Analysis, Triple Constraints, Radar Diagram

\section{INTRODUCTION}

Risk management is a vital and imperative matter to project managers; if not properly managed, risks may cause project failure (Royer, 2000). Risk is considered to be a major factor that influences project success and Project Risk Management (PRM) is an important process in any capital project (Krane et al., 2010), particularly construction projects. Thus, PRM is currently one of the main topics of interest for both researchers and construction practitioners (Raz et al., 2002). The Project Management Institute (PMI) is one of the standard-setting bodies for research in the PRM area; PMI shares and standardizes various available approaches. Furthermore, PMI recognizes PRM as a systematic and structured process for identifying and analyzing of risks, preparing a risk response and monitoring and controlling the response throughout the course of a project (PMI, 2009).
Of these constituent elements, one of the most important is risk analysis. Despite recognizing the importance and utility of an intuition-based "experimental system" of risk analysis (Slovic et al., 2004), it is still crucial to use algorithms and normative rules to attempt to create an "analytic system" (PMI, 2009). In particular, the analysis of risky situations in construction projects is a critical challenge for any construction project manager and further critical is the selection of an appropriate technique to assist project risk analysis (Baloi and Price, 2003). In fact, there is no universally accepted way to assess risks in all projects; the literature is full with a number of techniques (Dikmen et al., 2008), all claiming to be mathematically, statistically and from an engineering point of view, extremely competitive and effective. However, choosing the most suitable risk analysis technique for given project characteristics is critical to project success.

Corresponding Author: Alberto De Marco, Department of Management and Production Engineering, Politecnico di Torino, Turin, Italy 
In an attempt to help project managers choose the appropriate project risk analysis technique, this study proposes a framework methodology for selecting a specific qualitative or quantitative risk analysis technique under given characteristics of the project being managed.

This study is structured as follows: First, four main risk drivers are defined to capture and characterize the dimensions of a project; second, a number of risk analysis techniques are listed from literature; then, the methodology of selecting the appropriate risk analysis techniques by matching the main risk dimensions of a project is presented; and finally, after providing examples of application and discussion of the methodology, implications and conclusions are drawn.

\section{PROJECT DRIVER DESCRIPTIONS}

Tacit knowledge dictates to use specialized techniques where needed: High-risk projects require more sophisticated techniques and resources as contrast to small, low-risk projects (Ward, 1999). Such as, it seems reasonable to assign the experienced project managers at large, high-risk projects. Also, high-risk projects should be more carefully planned, closely monitored and strictly controlled (Couillard, 1995). In other words, a high level of risk requires a scrupulous project risk analysis and the best risk management techniques vary widely according to project characteristics.

A project may be characterized by a number of important drivers (dimensions), where each driver underlines a significant feature of the project. The traditional triple constraint (time, cost and quality/scope) of projects has already been proven to be inadequate (Norrie and Walker, 2004) and work has been conducted on determining additional and robust project dimensions (Shenhar et al., 1997). Moving forward with the detailed work of Pich et al. (2002) indirectly, it is proposed that a project can be described as driven by the following four main dimensions (or drivers), represented on the four axes of the radar diagram: Its level of challenge, the responsibility of the PM considering the size of the scope of work, the focus on one or more phases of its life-cycle and the level of maturity of the project management processes of the PM organization. The combination of these four factors allows one to conveniently frame the project into objective drivers of project risk. For example, a highly complex and large-sized project is likely to bring a high level of risk, which requires sophisticated risk analysis techniques.

The four abovementioned project drivers are discussed in further detail in following parts.

\subsection{Challenge}

Every project is a challenge and requires certain competencies for effective execution (Lampel, 2001). A broad range of technological or otherwise attributes define the level of challenge of a project, such as technological difficulty of task performance, differentiation and interdependency of operations, e.g., overlapping design and construction (Baccarini, 1996).

Here, the concept of being challenged with complexity in a project encompasses these definitions, with four levels of increasing challenge/complexity that can characterize a project (Shenhar and Dvir, 2007): (1) the uniqueness of the constructed facility; (2) the innovation of the building technology or of the construction process; (3) the complexity of the system design and its subsystem assemblies; and (4) the criticality of the time frame requiring a fast pace and time-critical construction effort. Therefore, a highly complex construction project can be a unique, complicated system design that uses breakthrough technology and requires a rapid development process.

\subsection{PM Responsibility}

Project scope, along with other key aspects, is a crucial stage, where risks associated with the project are analyzed and the specific project execution approach is defined (Ward, 1999). The success of a project is highly dependent on the level of effort expended during this scope definition phase (Cho and Gibson, 2001) and the scope size is an important factor influencing the number and impact of risks on a project.

The project scope and the associated inherent risk can be measured via four escalating factors: (1) the number of tasks required to accomplish the project; (2) the number of resources assigned to the tasks; (3) the magnitude of the budgeted/actual cost; and (4) the financial stress of the project's cash flow. Thus, a largesized project will have a large number of tasks with many assigned resources which results in a huge budget with deep financial exposure that demands anticipated equity capitals (Miller and Lessard, 2001).

\subsection{Focus}

It is important to consider the purpose and coverage of the management effort before managing a project because it allows an understanding on whether focus is needed at a single stage of the project or goes throughout the full project lifecycle. The process implies a notion of gradually increasing detail and focus on the provision of the final deliverable. This, in turn, may prove to be instrumental in addressing the inherent uncertainty attached to the 
fundamental question of 'what and how much to be done (Ward and Chapman, 2003)?'

A project can be addressed to cope with the following four main focuses, ranging from limited to extended: (1) proposal preparation during a bidding process; (2) either pure design or sole construction; (3) integrated design and construction and (4) lifecycle, which is usually a combination of the first three stages, namely design, construction and operations (Arditi and Gunaydin, 1998). A lifecycle focus is likely to bear more associated risks.

\subsection{Maturity}

PM organizations that undertake projects are always required to improve and adjust their operations and processes to plan, manage and complete projects more successfully due to constant pressure on project managers to integrate, plan and control complex projects (Ibbs and Kwak, 2000). Project organizations are exposed to maturity models of various types (Grant and Pennypacker, 2006). Furthermore, not only does the organizational project management maturity matter but also the risk management maturity of the PM organization.

PMI (2004) identifies the organizational project management maturity on four scales: (1) standardize the process; (2) measure the effectiveness of the standardized business processes in achieving desired outcomes; (3) control the developed processes, plans and implementations to achieve stability and (4) continuously improve by identifying new problems and implementing improvements to attain sustainability.

\section{PROJECT RISK ANALYSIS TECHNIQUES}

The process of project risk analysis demands appropriate and efficient techniques. A technique is a specific procedure designed to perform an activity or to solve a problem under a prescribed notation and guidelines (Brinkkemper, 1996).

The application of a risk analysis technique is often supported by tools that can be automated. The main role of the tools is to allow for searching, gathering and managing the necessary data for the various PRM phases. Various techniques use different types of data and information collected from a wide range of sources using different tools, such as statistics, inspections, surveys, documentations and expert judgments (Gilbert, 1989).

Project risk analysis techniques can be classified into two main categories, namely qualitative and quantitative techniques (PMI, 2009), with associated sub-categories of semi-quantitative and simulation techniques. The group of qualitative risk analysis techniques does not operate on numerical data, presenting results in the form of descriptions, recommendations and ordinal scores (Hubbard and Evans, 2010), where risk assessment is connected with qualitative description and determination of qualitative scales for the probability and impact of the consequences of risk. Qualitative techniques can be lists of risks, risk rankings, or risk maps. These techniques prioritize risks for subsequent further analysis or action by assessing and combing their probability of occurrence and impact. The risk is evaluated in more conceptual terms, such as high, medium or low, depending on the collected opinions and risk tolerance boundaries in the organization.

The main qualitative analysis techniques are: Brainstorming: Best possible solutions of project risk are generated and determined under the leadership of a facilitator (Berg, 2010); cause and effect diagram: Also known as the Ishikawa or fishbone diagram, it is useful for identifying and analyzing causes of risks (Del Cano, 2002); checklists: A detailed aide-memoire for the identification of potential risks based on past similar projects (Del Cano, 2002); delphi: A facilitator uses a questionnaire to solicit ideas about the major project risks and project risk experts participate anonymously (Berg, 2010); Event Tree Analysis (ETA): Models the range of possible outcomes of one or a category of initiating events and usually provides qualitative descriptions (Del Cano, 2002); Risk Breakdown Matrix (RBM): An 'activities and threats' matrix, where the risk number for each activity and the most frequent overall risks are evaluated (Hillson et al., 2006); risk data quality assessment: Evaluates the extent to which a risk is understood and the accuracy, quality, reliability and integrity of the risk data (PMI, 2009).

A derivative group of techniques is the one that uses a semi-quantitative assessment of risk. Semi-quantitative analysis can be defined when a scale factor is associated to nonnumeric rankings.

Some of the semi-quantitative variants of qualitative techniques are: Interviewing: Risks are identified through expert interviews and a risk management capability score is determined with a five-point scale. This technique is also used to assess the probability and impact of risks on project objectives (IMA, 2007); risk mapping, risk matrix, probability and impact matrix: Used to semi-quantitatively evaluate and prioritize a group of risks that could significantly impact the project cost and time outcomes (Scandizzo, 2005); risk probability and impact assessment: Investigates the likelihood and potential effect of a risk on projects objectives (PMI, 2009). 
With quantitative analysis techniques, the estimation of risk exposure is related to the application of numerical measures. Here, the impact of consequences is defined as a monetary value and the likelihood by the frequency of risk occurrence based on past series of available data. In brief, quantitative techniques numerically analyze the effect of identified risks on the project objectives (PMI, 2009).

The main quantitative techniques are: Decision tree analysis: A decision flow diagram subject to the influence of future events with a known probability of occurrence (Schuyler, 2001); expected monetary value: Takes into consideration the probability aspect of the system states and is based on a gain matrix (PMI, 2009); expert judgment: Based on expert opinions to evaluate the failure rate and success chances of the overall project (PMI, 2009); Fault Tree Analysis (FTA): Possible derivative risk events are derived from a top event (Del Cano, 2002); fuzzy logic: A simple way to reach a definite conclusion based on vague, imprecise, noisy or missing input (Konstandinidou et al., 2006); probability distributions: Continuous probability distributions represent the uncertainty in values, such as durations of schedule activities and costs of project components (Del Cano, 2002; PMI, 2009); sensitivity analysis/tornado diagram: Helps to determine which risks have the greatest potential impact on the project. Using a Tornado diagram, an attempt is made to capture how much risk impacts a particular metric, such as revenue or earnings (Lyons and Skitmore, 2004).

In addition, risk analysis techniques that use computer-based simulation tools, such as Monte Carlo simulations and system dynamics applications for PRM, can be considered derivative concepts of quantitative analysis techniques because of the extended use of numerical past data for risk analysis. Simulations are of great value when large sets of historical data from past projects are available.

Some of the techniques in this category are: Monte Carlo: Evaluates decisions related to future events that can be described with probabilistic distributions. Monte Carlo simulations randomly choose values for uncertain variables to generate a distribution of possible case scenarios (De Marco, 2011); system dynamics: Allow for diagramming a system of causally looped variables, defining the mathematical relations and instructing a computer to solve the differential set of equations with the purpose of assessing the impact on project performance.

\section{METHODOLOGY}

The four categories of risk analysis techniques can be plotted according to their degree of analytic assessment of risk exposure, from qualitative analysis to simulation. It can be argued that quantitative and particularly simulation-based techniques require a larger effort to gather and process data compared with qualitative assessment techniques. Consequently, quantitative techniques are likely to be applied in projects with a greater level of risk. The idea is graphically demonstrated in Fig. 1, where the four categories of risk analysis techniques are incorporated with the four project drivers discussed in previous sections.

Plotting the project drivers on the chart results in determining the risk analysis technique category; this is suitable for the given variables.

It is imperative to mention that all projects do not necessarily demonstrate a 'balanced' feature on the radar diagram: Certain dimensions may be more skewed and stretched than others, rendering a poorly adjusted and unbalanced diagram. In such situations, it is always advisable to consider at least the plotted area (category of risk analysis technique) covered by joining all four ends. Furthermore, a middle ground is suggested as a compromise because the dominance of 'Maturity' must be considered because a less mature PM organization might not be in a comfortable position to use sophisticated techniques. Therefore, an informed 'subjective' decision may be made in such cases.

In other terms, the extension of the project plotted area on the four dimensions diagram is an indicator of the extent to which quantitative techniques might beneficially apply to the risk analysis process.he radar diagram.

\section{DEMONSTRATION PROJECTS}

To practically demonstrate the applicability of the proposed methodology, reported below are two projects from the direct experience of the authors involved as construction consultants/experts. The projects were selected based on the differences in their radar diagram, which provides a better understanding of how to select a certain category and/or meet halfway.

\subsection{University Campus Project}

\subsubsection{Project Overview}

This first sample project is about the development of a new educational facility in the city center of Turin, Italy (UDST, 2009). 


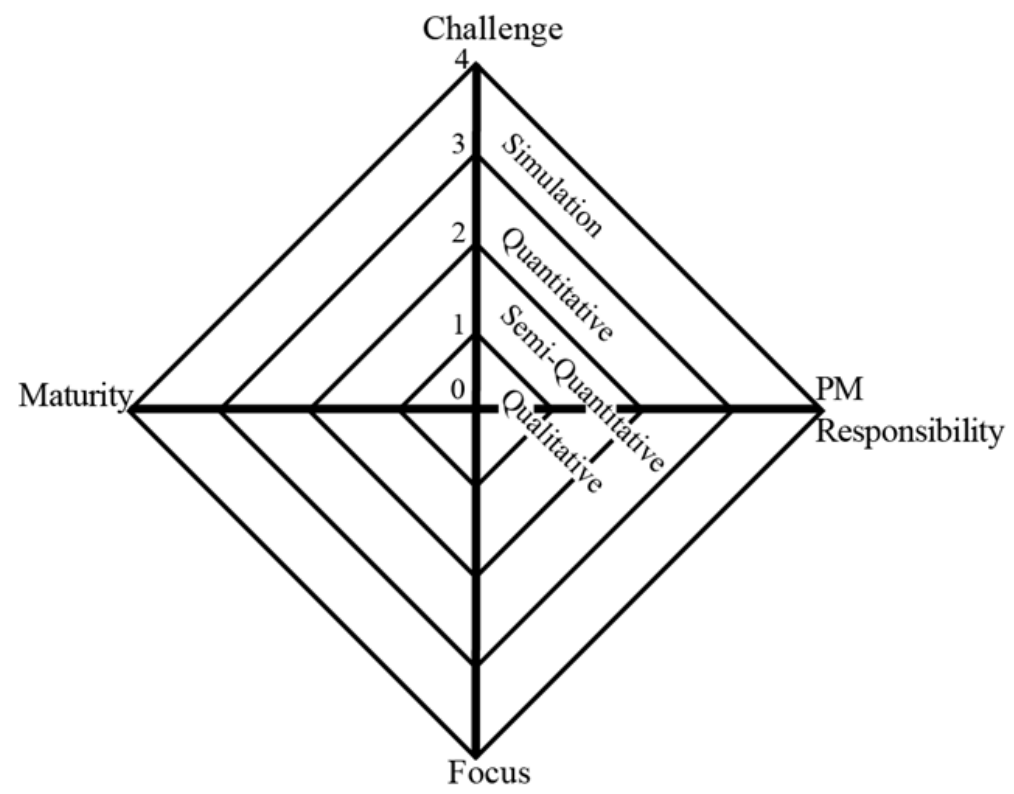

Fig. 1. Categories of risk analysis techniques incorporated in the radar diagram

In 2006, the university engaged the service of a Build-Operate-Transfer concessionaire to design, secure permits, finance, build and maintain the facility, which consists of more than 17,000 square meters of above ground functions (lecture rooms, office buildings, dormitories, kindergartens, support stores, commercial services) and more than 20,000 square meters of underground facilities (parking lots, a gym and a swimming pool). The private investment of $€ 40$ million will be reimbursed via an annual unitary charge payment by the university, including all facility management services and rental fees obtained from operating the commercial and parking functions. An initial public funding of approximately $€ 6$ million is made available.

\subsubsection{Project Risks}

Here is a short description of what happened and the major obstacles incurred during the course of action. The project, up to May 2012, has undergone various major risks, namely, design changes due to varied rental market conditions following the 2007/08 real estate crisis; the design not being approved by the fire protection agencies due to design changes; archeological discoveries in the underground excavations; financial stress due to the 2010/11 credit crunch with escalation in interest rates; financial problems of the leading company; no equity available to fund the design period up to the financial closure and ground breaking; an experienced project manager quitting at a crucial stage of the design development; level of bankability lower than expected; changes in the pre-agreed term sheet of financial closure; increase in unexpected financial closure transactional costs; lack of investment funds interested in entering the SPV capital; financial problems of the university; changes in the BOT system regulation in Italy; underperforming commercialization/lower level of expected market revenue; difficulty in finding interested gym and swimming pool operators; and increased level of dispute between the project partners.

\subsubsection{Project Mapping on the Radar Chart}

The project is identified on the anticipated radar diagram's axes (Fig. 1) as follows: Challenge 3, PM Responsibility 4, Focus 4 and Maturity 1. The medium-high level of challenge is due to the interconnection of various systems and buildings devoted to the different functions. However, the project is neither time critical nor at a high level of innovation in building technology and process.

Because the project is a privately financed public facility, the Special Purpose Vehicle (SPV) company has tremendous pressure on the cash stream, which becomes extremely critical when the financial closure is secured with the banks' pool. The focus is on the total BOT life cycle from the initial concept design to operations. Finally, the medium-sized family owned company, 
which holds $75 \%$ of the SPV and associated project's design and construction efforts, is at a low level of maturity in the project management because there are not refined methods for measuring, controlling or continuously improving the performance of their project management processes. The project drivers are plotted on the radar diagram in Fig. 2. The project poses an 'unbalanced' mapping on the radar chart. It suggests that quantitative techniques be used, not a simulation due to the level of challenge. However, the extremely low level of maturity results in an even lower sophisticated category of techniques, which is more aligned with acceptance/expertise and usage. However, using only qualitative techniques would be too simplistic and unjustified: Aligned with the level of maturity but not adequate for high levels of other drivers. Thus, it is proposed to use the semi-quantitative category and it is further proposed to use the "Probability-Impact Matrix" technique.

\subsubsection{Risk Analysis}

In Table 1, the risk analysis is performed using the data of the project during the year 2008 with the help of the selected technique. Also, based on the "Probability and Impact Risk Ranking" (PMI, 2009), the risks have been categorized into 'High', 'Medium' or 'Low' risks.

\subsubsection{Discussion}

The use of a simple and less sophisticated technique results in a less than thorough but at least, a guaranteed risk analysis, which otherwise could be ignored. Additionally, the conventional risk ranking serves the purpose of risk analysis because it draws the management attention towards critical aspects of the project, which if mitigated and managed intelligently, will result in a higher probability of project success. Apparently, it seems evident from the current scenario that the most serious risks of the project were actually the ones with high ranks obtained from the analysis. Therefore, it can be safely assumed that the technique selected using the radar diagram given the project drivers proved to be apt and sufficient for the risk analysis.

The project manager, when requested to provide feedback of this analysis, concluded that the methodology is viable and useful in selecting risk analysis techniques that are suitable for the level of the project complexity and maturity of the project environment. The project manager stated that this methodology does not provide an unnecessary managerial burden to the project management duty. On the contrary, the methodology helps to provide the right tools for the right project.

Table 1. Risks and application of the semi-quantitative Probability-Impact Matrix technique

\begin{tabular}{lllll} 
Description & Prob. & Impact & $\begin{array}{l}\text { What } \\
\text { occurred }\end{array}$ & $\begin{array}{l}\text { Risk } \\
\text { rank }\end{array}$ \\
\hline $\begin{array}{l}\text { Design changes due to varied } \\
\text { rental market conditions }\end{array}$ & 2 & 5 & Market crisis \\
$\begin{array}{l}\text { Design not approved by permit authorities } \\
\text { Archeological discoveries in the }\end{array}$ & 1 & 4 & $\begin{array}{l}\text { Firemen } \\
\text { Unknown 9th century walls }\end{array}$ \\
$\begin{array}{l}\text { underground excavations } \\
\begin{array}{l}\text { Increase in interest rates } \\
\text { charged by lending institutions }\end{array}\end{array}$ & 4 & 2 & L \\
$\begin{array}{l}\text { Financial problems of the } \\
\text { learg }\end{array}$ & 3 & 5 & World credit crunch \\
Bankruptcy
\end{tabular}

leading company. No equity available

to fund the design period up to the

financial close/ground breaking.

Project manager turnover

Liquidity due to crisis

14 Quit when company started having problems

$\mathrm{L}$

Pre-agreed term sheet of financial close changed. Increase in unexpected

financial close transactional costs (business planning, banks' due diligence).

No investment fund interested in entering the SPV capital.

Granting authority's budget cuts

Financial problems of the university meant incapable of paying

Changes in the BOT

system regulation in Italy

Revenue/market risk

$4 \quad 3$

the additional annual charge to assure the project's bankability

32 The project was not affected

24 Underperforming commercialization/lower level of market revenue.

Unable to find an interested gym and swimming pool operator due to the $\quad \mathrm{M}$

financial crisis and high level of fees demanded by the

business plan to assure profitability

Stakeholders' dispute

$2 \quad 5 \quad$ Increased level of dispute between the project's partners

$\mathrm{H}$

Probability Scale: 1- Very Low, 2- Low, 3-Medium, 4-High, 5-Very High; Impact Scale: 1-Very Low, 2-Low, 3- Medium, 4- High, 5- Very High; Risk Rank Scale: H-High, M-Medium, L-Low 


\subsection{Container Yard and Quay Wall Expansion Project}

\subsubsection{Project Overview}

$\mathrm{T}$ This second sample project is a port expansion project, where the capacity of the existing container terminal in the port city of Karachi, Pakistan was increased. In 2005, Karachi International Container Terminal (KICT), a member of the Hutchison Port Holdings Group, which has been enjoying the support and expertise of the world's leading port investor, developer and operator to help transform KICT into a major container handling facility that is capable of receiving the region's increasing container trade, entered into an agreement with the Karachi Port Trust (KPT) for the development of its Phase III project at West Wharf of Karachi Port (KPT).

In addition to extending the existing concession period, the project involved deepening the alongside draft to 14 meters; increasing the handling capacity by acquiring and redeveloping additional land area; and acquiring additional quayside and container yard equipment. Before the Phase III expansion, operational terminal area was 135,122 sqm, length of berths was 500 $\mathrm{m}$ with an annual capacity of 400,000 TEUs at an initial capital cost of US\$ 65 million.

BThe purpose of the projected expansion was to increase the terminal to $260,000 \mathrm{sqm}$, berths length to $973 \mathrm{~m}$ and annual capacity to 700,000 TEUs with an additional investment of US\$ 55 million. Additionally, the berths of the terminal were deepened to allow a 14meter draught container ship.

\subsubsection{Project Risks}

The project underwent a variety of major, negative events/risks, namely the following: Design changes in the length of the pile driving due to the varied geotechnical conditions on site; unexpected and uneven settlement of the berth surface adjacent to the pile driving site, which rendered almost half of the old berth area unusable; and financial stress due to the 2008 credit crunch worldwide crisis with an increase in the interest rates charged by the lending institutions.

\subsubsection{Project Mapping on the Radar Chart}

The project may be plotted on the radar diagram as follows: Challenge 3, PM Responsibility 3, Focus 3 and Maturity 2. The medium-high level of challenge is due to the complex interconnection of various systems, structures and buildings devoted to different functions.

Although the project is not time critical, it possesses a medium level of innovation in building technology and process. Due to private finance and sophisticated governmental associations, the PM Responsibility is limited to the management of the budget only, where cash flow was primarily taken over by the client. The focus is on the total BOT life cycle from the initial concept design to operations. Finally, though large-sized organizations were involved, their maturity was limited to only measuring their project management processes. The project drivers are plotted on the radar diagram shown in Fig. 3.

The mapping of the current project is less 'unbalanced' compared with that of the previous project (Fig. 2). The category of simulation techniques cannot be considered because there is only one driver (Focus) plotted in that region. Moreover, the categories of quantitative and semiquantitative techniques seem a bit too simplistic given the overall complexity posed by the current project. Therefore, the natural choice would be quantitative techniques, which can be narrowed down to the "Decision Tree Analysis" technique. Due to space limitations, only the first risk ('design changes') is analyzed to demonstrate the applicability of the methodology. Additionally, established by later events, this risk proved to be extremely critical and was an enormous nuisance.

\subsubsection{Risk Analysis}

The project design team had an ambitious plan when they decided to opt for driving the steel tubular piles to support the existing quay wall to deepen the available draft. Although there was the possibility of in-situ construction, the new design was much too alluring and the associated risks were ignored. This analysis considers the possible alternatives and related probabilities.

The design could be either driving the piles or in-situ construction. Furthermore, there was considerably large probability of the piles reaching the design depth; however, in case they did not, although the probability was low, the impact was much higher in terms of monetary value, as shown in Fig. 4. Therefore, two remedial actions could be taken: Either doing the partial excavation to reach the design depth and pouring concrete to fill the gap or removing the piles and constructing the piles in-situ.

\subsubsection{Discussion}

The use of quantitative techniques ensures a better and more reliable analysis in this case. Furthermore, remembering the type of risk, a decision tree analysis seems to be an appropriate choice. From the project manager's interview and other sources of information, it was found that due to geotechnical conditions, which were not properly investigated before the design, the design could not be realized and additional steps were taken that caused major delays and budget overruns in the project. 


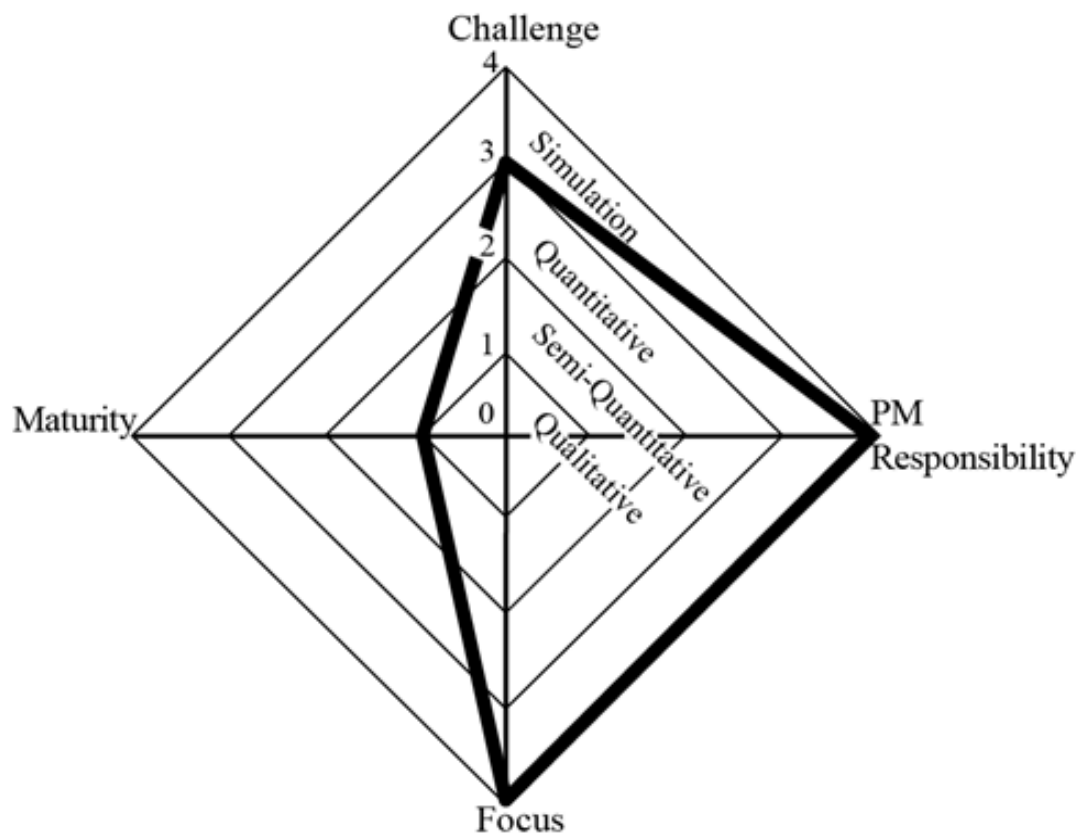

Fig. 2. Dimensions of the university campus project on the radar diagram

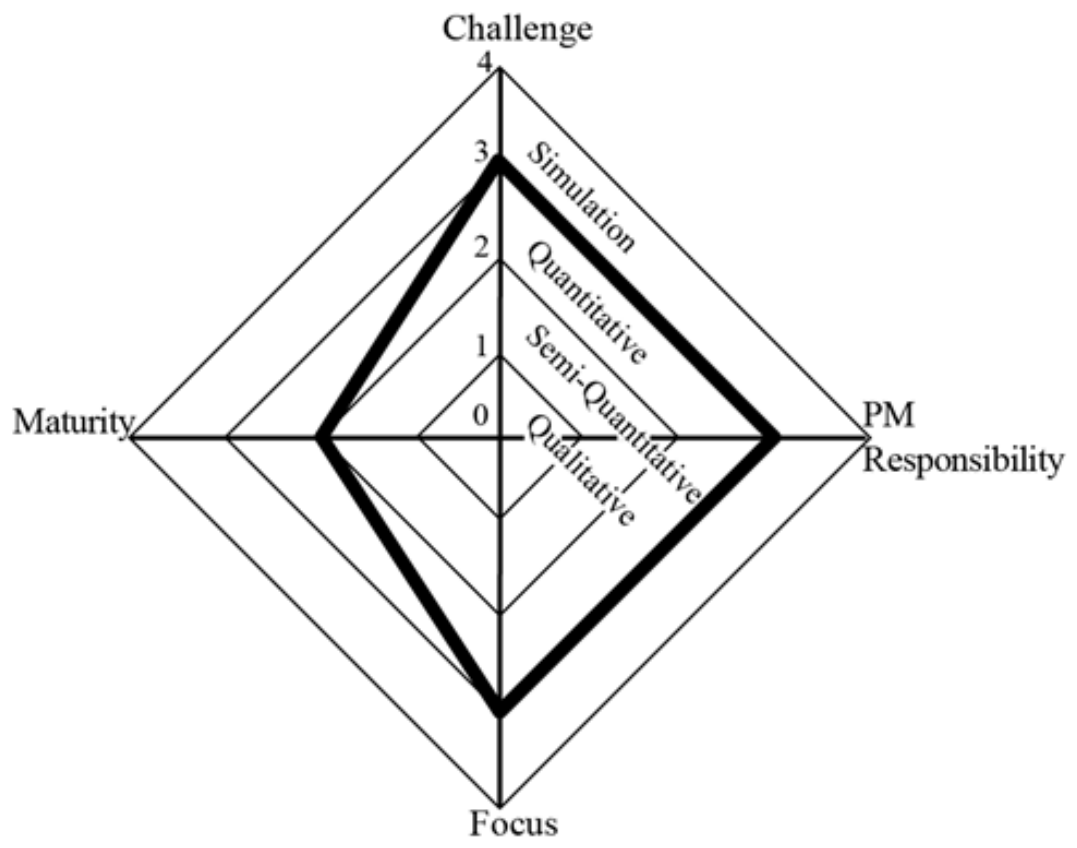

Fig. 3. Dimensions of the Container Yard and Quay Wall expansion project on the radar diagram

Therefore, the present scenario suggests that the category and technique selected by the radar diagram with the help of project drivers sufficiently addresses the risk analysis.

AJAS 


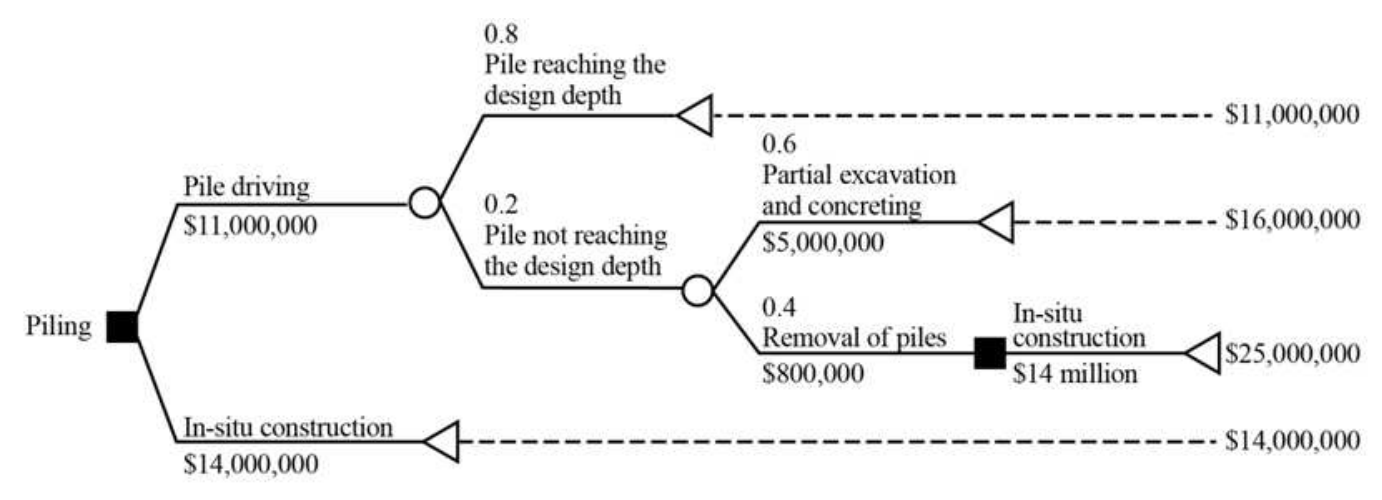

Fig. 4. Decision tree analysis of the 'Design changes' risk in the port expansion project

The project manager was further requested to provide a feedback of this ex-post analysis. Based on his comments, it can be confidently concluded that the accuracy and efficiency of the framework methodology left a satisfactory impression and the project manager was interested about the use of the proposed methodology.

\section{LESSONS LEARNED AND IMPLICATIONS}

With increased research and development efforts in the area of construction PRM, a larger variety of tools and techniques to help perform risk management and improved performance of those tools and techniques, it becomes extremely important for project managers to select the appropriate tool or technique. To ascertain the suitability of the risk analysis technique, important, select drivers of the project are suggested to be used in a graphical manner. By carefully plotting and interpreting the radar chart, an advisable category of risk analysis techniques can be reached, followed by subsequently choosing a technique.

Furthermore, the use of the resulting technique will aim to sufficiently reach the required sophistication and reliability of the results. The technique will save the project managers from investing too little or too much effort and money for the risk analysis activity, ensuring a productive use of resources. It does not matter the type, size or the final budget of the construction project to create the radar diagram; the only required information is the level of its drivers-similar techniques can be obtained for projects with different budgets.

Finally, this study attempts to attract the consideration of construction management research and practitioner community to help further the applicability, suitability and affectivity of this methodology at a larger level so the testimony of consulted project managers can be justified.

\section{SUMMARY}

Complex projects require more sophisticated risk analysis techniques and vice versa: The cost and effort involved in performing expensive and labor-exhaustive analysis using simulations will benefit only when it is required, e.g., on complex, exceptional and rare projects. Simpler and routine projects may benefit from relatively simpler analysis techniques, such as qualitative techniques.

This study presents a practical methodology for helping project managers select the appropriate risk analysis technique. The methodology also broadens the perspective of the project drivers from conservative triple constraints (i.e., time, cost and quality/scope) to more extensive and realistic constraints (i.e., complexity, size, focus and maturity).

The methodology is then applied to two construction projects by creating the proposed radar diagram, obtaining the suitable category of techniques, selecting an appropriate technique from the collected depository of techniques and performing an ex-post risk analysis. The results and feedback from the associated project managers seem promising and call for more exhaustive testing at a broader level to ascertain the universality of the proposed methodology in the construction industry.

\section{REFERENCES}

Arditi, D. and H.M. Gunaydin, 1998. Factors that affect process quality in the life cycle of building projects. J. Construct. Eng. Manage., 124: 194-204. DOI: 10.1061/(ASCE)0733-9364(1998)124:3(194) 
Baccarini, D., 1996. The concept of project complexity-a review. Int. J. Project Manage., 14: 201-204. DOI: 10.1016/0263-7863(95)00093-3

Baloi, D. and A.D.F. Price, 2003. Modelling global risk factors affecting construction cost performance. Int. J. Project Manage., 21: 261-269. DOI: 10.1016/S0263-7863(02)00017-0

Berg, H.P., 2010. Risk management: Procedures, methods and experiences. Risk Manage., 1: 79-95.

Brinkkemper, S., 1996. Method engineering: Engineering of information systems development methods and tools. Inform. Software Technol., 38: 275-280. DOI: 10.1016/0950-5849(95)01059-9

Cho, C.K. and G.E. Gibson, 2001. Building project scope definition using project definition rating index. J. Archit. Eng., 7: 115-125. DOI: 10.1061/(ASCE)1076-0431(2001)7:4(115)

Couillard, J., 1995. The role of project risk in determining project management approach. Project Manage. J.

De Marco, A., 2011. Project Management for Facility Constructions. 1st Edn., Springer, Berlin, ISBN-10: 3642170927, pp: 197.

Del Cano, A., 2002. Integrated methodology for project risk management. J. Construct. Eng. Manage., 128: 473-486. DOI: 10.1061/(ASCE)07339364(2002)128:6(473)

Dikmen, I., M.T. Birgonul, C. Anac, J.H.M. Tah and G. Aouad, 2008. Learning from risks: A tool for postproject risk assessment. Automat. Construct., 18: 42-50. DOI: 10.1016/j.autcon.2008.04.008

Gilbert, I.E., 1989. Guide for Selecting Automated Risk Analysis Tools. 1st Edn., National Institute of Standards and Technology, Gaithersburg, ISBN-10: 0160002966, pp: 26.

Grant, K.P. and J.S. Pennypacker, 2006. Project management maturity: An assessment of project management capabilities among and between selected industries. IEEE Trans. Eng. Manage., 53: 59-68. DOI: 10.1109/TEM.2005.861802

Hillson, D.A., S. Grimaldi and C. Rafele, 2006. Managing project risk using a cross risk breakdown matrix. Risk Manage., 8: 61-76. DOI: 10.1057/palgrave.rm.8250004

Hubbard, D. and D. Evans, 2010. Problems with scoring methods and ordinal scales in risk assessment. IBM J. Res. Dev., 54: 2-10. DOI: 10.1147/JRD.2010.2042914
Ibbs, C.W. and Y.H. Kwak, 2000. Assessing project management maturity. Project Manage. Inst., 31: 3243.

IMA, 2007. Enterprise Risk Management: Tools and Techniques for Effective Implementation. Statements on Management Accounting, Institute of Management Accountants.

Konstandinidou, M., Z. Nivolianitoub, C. Kiranoudisa and N. Markatos, 2006. A fuzzy modeling application of CREAM methodology for human reliability analysis. Reliab. Eng. Syst. Safety, 91: 706-716. DOI: 10.1016/j.ress.2005.06.002

Krane, H.P., A. Rolstadas and N.O.E. Olsson, 2010. Categorizing risks in seven large projects-Which risks do the projects focus on? Project Manage. J., 41: 81-86. DOI: 10.1002/pmj.20154

Lampel, J., 2001. The core competencies of effective project execution: The challenge of diversity. Int. J. Project Manage., 19: 471-483. DOI: 10.1016/S02637863(01)00042-4

Lyons, T. and M. Skitmore, 2004. Project risk management in the Queensland engineering construction industry: A survey. Int. J. Project Manage., 22: 51-61. DOI: 10.1016/S02637863(03)00005-X

Miller, R. and D. Lessard, 2001. Understanding and managing risks in large engineering projects. Int. J. Project Manage., 19: 437-443. DOI: 10.1016/S02637863(01)00045-X

Norrie, J. and D.H.T. Walker, 2004. A Balanced scorecard approach to project management leadership. Project Manage. J., 35: 47-56.

Pich, M.T., C.H. Loch and A. De Meyer, 2002. On uncertainty, ambiguity and complexity in project management. Manage. Sci., 48: 1008-1023. DOI: 10.1287/mnsc.48.8.1008.163

PMI, 2004. Organizational Project Management Maturity Model OPM3. 1st Edn., Project Management Institute, Newtown Square, ISBN-10: 1930699042, pp: 42.

PMI, 2009. Practice Standard for Project Risk Management. 1st Edn., Project Management Institute, Newtown Square, ISBN-10: 193389038X, pp: 116.

Raz, T., A.J. Shenhar and D. Dvir, 2002. Risk management, project success and technological uncertainty. R\&D Manage., 32: 101-109. DOI: 10.1111/1467-9310.00243 
Royer, P.S., 2000. Risk management: The undiscovered dimension of project management. Project Manage. J., 31: 6-13.

Scandizzo, S., 2005. Risk mapping and key performance indicators in operational risk management. Econ. Notes, 34: 231-256. DOI: 10.1111/j.03915026.2005.00150.x

Schuyler, J.R., 2001. Risk and Decision Analysis in Projects. 2nd Edn., Project Management Institute, Newtown Square, ISBN-10: 1880410281, pp: 259.

Shenhar, A. and D. Dvir, 2007. Reinventing Project Management: The Diamond Approach to Successful Growth and Innovation. 1st Edn., Harvard Business School Publishing India Pvt. Limited, Boston, ISBN-10: 1591398002, pp: 276.
Shenhar, A.J., O. Levy and D. Dvir, 1997. Mapping the dimensions of project success. Project Manage. J., 28: 5-13.

Slovic, P., M. Finucane, E. Peters and D.G. MacGregor, 2004. Risk as analysis and risk as feelings: Some thoughts about affect, reason, risk and rationality. Risk Anal., 24: 311-322. DOI: 10.1111/j.02724332.2004.00433.x

UDST, 2009. Nuovo complesso polifunzionale. Aldo Moro. Ward, S.C. and C.B. Chapman, 2003. Transforming project risk management into project uncertainty management. Int. J. Project Manage., 21: 97-105. DOI: 10.1016/S0263-7863(01)00080-1

Ward, S.C., 1999. Requirements for an effective project risk management process. Project Manage. J., 30: 37-43. 